\title{
Andicolea, a replacement name for Loricaria (Asteraceae, Gnaphaliinae)
}

\author{
Luis Mayta ${ }^{1}$ \& Eduardo A. Molinari-Novoa2*
}

\section{Article info}

Received: 22 Nov. 2020

Revision received: 7 Jun. 2021

Accepted: 29 Jun. 2021

Published: 15 Jul. 2021

\section{Associate Editor}

Mike Thiv

\section{Introduction}

Loricaria Wedd. (1855) is a genus of Andean Asteraceae from the subtribe Gnaphaliinae (Smissen et al. 2020) characterised by distichous branches with densely imbricate coriaceous leaves that conceal the capitula when flowering (Hind 2004), making them a very recogniseable genus at high altitudes. It comprises twenty species (Cuatrecasas 1954; Dillon \& Sagástegui-Alva 1986; Hind 2004).

While creating a database for algal genera, the second author realised that Loricaria is a later homonym of Loricaria J.V. Lamour. (Bory de Saint-Vincent 1825), a genus based on Fucus loreus L. [=Himanthalia elongata (L.) Gray, Himanthaliaceae]. No proposal for its conservation has been made, despite the annotation in the entry of the genus in Flann (2020), nor has a replacement name been published. We therefore propose a new name, Andicolea, in reference to its Andean distribution.

\section{Nomenclatural history of Loricaria J.V. Lamour.}

Lamouroux (1813) proposed the division of the genus Fucus L. into eleven unnamed sections, adding that most of them would constitute proper genera, more natural and better characterised than those proposed by 'naturalistes modernes'.

\footnotetext{
${ }^{1}$ National University of Saint Augustine, Arequipa 04001, Peru. https:// orcid.org/0000-0001-8359-4876

2 Chess Consulting \& Project, Lima 15039 and 'La Molina' National Agrarian University, Lima 15024, Peru. https://orcid.org/0000-00032865-1211

* Corresponding author e-mail: eduardomolinov@gmail.com
}

In his entry on the 'Fucacées' in the Dictionnaire classique d'histoire naturelle (Bory de Saint-Vincent 1825), Lamouroux cites his 1813 essay in the very first sentence, as it is the main source of discussion, establishing an indirect reference to the validating descriptions in French and Latin [Art. 38.1(a) of the Shenzhen Code].

In the last paragraph, Lamouroux refers his (1813) section III to section V, section XI to section VII, and section $\mathrm{X}$ to Fucus sensu stricto, that is, his section VI. He then proceeds to list the accepted sections with their proper generic names in successive order, thus connecting the sections' descriptions with them: section I is Sargassum C. Agardh, section II is Turbinaria J.V. Lamour., section IV is Siliquaria Stackh., section V (which includes section III) is Cystoseira C. Agardh, section VI (which includes section X) is Fucus, section VII (which includes section XI) is Nodularia J.V. Lamour., section VIII is Moniliformia J.V. Lamour. and section IX is Loricaria. After that, Lamouroux lists the genera erected in his 1813 essay, finishing the entry.

Since Lamouroux (in Bory de Saint-Vincent 1825) repeatedly refers to his 'Essai', following its systematic treatment, with modifications from Stackhouse (1809) and Agardh (1821), Loricaria was validly published under the provisions of Art. 38.14 of the Shenzhen Code, as it were Turbinaria, Nodularia and Moniliformia. Therefore, Loricaria Weddell is an illegitimate later homonym under the provisions of Art. 53.1 of the Shenzhen Code.

\section{Taxonomic treatment}

Here, we propose new combinations in Andicolea for the twenty known species of Loricaria, under the sections 
proposed by Cuatrecasas (1954), listed alphabetically. The only subspecific taxon proposed in that same work that we transfer here is L. thujoides var. thyrsoidea, as Andicolea thyrsoidea, following Dillon \& Sagástegui-Alva (1986). We refrain from transferring the rest since most of them are based mainly or exclusively on the size and disposition of the leaves, a variable character in our opinion. One variety, namely L. thujoides var. stuebelii, has been already synonymized with its parent taxon (Rodríguez Rodríguez et al. 2016). As reported by Rangel Churio (2000), the isoneotype of Loricaria stenophylla var. microphylla at COL (Cuatrecasas 14692) gives more information about the locus typicus than what was written in the typification by Cuatrecasas (1954).

Andicolea Mayta \& Molinari, nom. nov.

Replaced synonym: Loricaria Wedd., Chlor. Andina 1: 165. 1855., non Loricaria J.V. Lamour. in Bory de Saint-Vincent Dict. Class. Hist. Nat. 7: 71. 1825.

$\equiv$ Tafalla D. Don, Edinburgh New Philos. J. 11: 273. 1831.

三 Baccharis ser. Distichae DC., Prodr. 5: 426. 1834.

Lectotype: Conyza thujoides Lam. (designated by Cuatrecasas, 1954: 154).

Etymology. The name is based upon New Latin andicola, from 'Andes' (declined in analogy with Alpēs) and 'colō', meaning 'inhabitant of the Andes'.

\section{Andicolea sect. Andicolea}

$\equiv$ Loricaria sect. Loricaria

$\equiv$ Loricaria sect. Thyopsis Cuatrec., Feddes Repert. Spec. Nov. Regni Veg. 56: 154. 1954.

Type: Conyza thujoides Lam., Encycl. [J. Lamarck \& al.] 2: 90.1786.

Andicolea azuayensis (Cuatrec.) Mayta \& Molinari, comb. nov.

Basionym: Loricaria azuayensis Cuatrec., Feddes Repert. Spec. Nov. Regni Veg. 56: 158. 1954.

Type: Ecuador, Azuay: páramos in vicinity of Toreador, between Mollcturo and Quinoas, 3810-3930 m, 15 June 1943, J. A. Steyermark 53210 (F - holotype [photo!]; RB, G - isotypes).

Notes. Andicolea azuayensis is endemic to Ecuador, it is considered an Endangered species (León Yáñez et al. 2011; Ulloa Ulloa et al. 2017).

Andicolea cinerea (D.J.N. Hind) Mayta \& Molinari, comb. nov.

Basionym: Loricaria cinerea D.J.N. Hind, Kew Bull. 59(4): 541. 2004.

Type: Ecuador, Azuay: Cordillera occidental, Laguna Luspa, $20 \mathrm{~km} \mathrm{~W}$ of Cuenca, El Cajas National Reservation Area, 3800 m, 19 Aug. 1985, Ramsay \& Merrow-Smith 122 (K holotype [photo!]; QCNE - isotypes).

Notes. Andicolea cinerea is endemic to Ecuador (Ulloa Ulloa \& Neill 2005; Ulloa Ulloa et al. 2017). It is distinguished from other species of the genus by its dense pubescence (Hind 2004).
Andicolea complanata (Sch. Bip.) Mayta \& Molinari, comb. nov.

Basionym: Baccharis complanata Sch. Bip., Bonplandia 4: 51.1856.

$\equiv$ Loricaria complanata (Sch. Bip.) Wedd., Chlor. Andina 1: 167.1855 .

Type: Colombia, Santander: páramos de Santurbán, September 1845, Purdie [s.n.] (K - lectotype [photo!], here designated, see the notes. K, P - isolectotypes [photo!]). Colombia, Norte de Santander: Pamplona, páramos de Santurbán, Linden 720 (P, BR - syntypes [photo!]). Colombia, Norte de Santander: Pamplona, páramos de Santurbán, Funk \& Schlim 1292 (P, BR - syntypes [photo!]).

Notes. Andicolea complanata grows in the paramo phytocoenoses of Colombia and Ecuador (León Yánez et al. 2011; Bernal et al. 2016; Ulloa Ulloa et al. 2017). Cuatrecasas (1954) selected the collection of Purdie as lectotype, without seeing it. We here designate the sheet in Kew (barcode K000222173), in better shape than its duplicates, as a second-step lectotype, narrowing his selection.

Andicolea lagunillensis (Cuatrec.) Mayta \& Molinari, comb. nov.

Basionym: Loricaria lagunillensis Cuatrec., Feddes Repert. Spec. Nov. Regni Veg. 56: 162. 1954.

Type: Colombia, Cauca: Cordillera Central, cabeceras del río Palo entre San Paulino y López, páramos de la Lagunilla en Las Casitas, 3700 m, 3 December 1944, R. Cuatrecasas 18988 (F-holotype, isotype [photo!]; A, COL, DUKE, GH, K, MICH, MO, NY, P - isotypes).

Notes. Andicolea lagunillensis can be found in the paramo phytocoenoses of Colombia (Bernal et al. 2016; Ulloa Ulloa et al. 2017).

Andicolea leptothamna (Mattf.) Mayta \& Molinari, comb. nov.

Basionym: Tafalla leptothamna Mattf. Notizbl. Bot. Gart. Berlin-Dahlem 10: 775. 1929.

Lectotype (designated here): Peru, La Libertad: Pataz, cerros entre Tayabamba y el valle del río Mixiollo, 3600 m, 02 August 1914, A. Weberbauer 7027 (MOL - lectotype!, F, GH, US - isotypes).

$\equiv$ Loricaria leptothamna (Mattf.) Cuatrec. Feddes Repert. Spec. Nov. Regni Veg. 56: 163. 1954.

Notes. Since the holotype could not be found in the Berlin herbarium (León 2002), we designate the complete duplicate (barcode MOL00006609) in a Peruvian herbarium as lectotype, conforming to current Peruvian law. The species has been found also in Ecuador (León et al. 2006; Neill \& Ulloa Ulloa 2011; Ulloa Ulloa et al. 2017).

Andicolea ollgaardii (M.O. Dillon \& Sagást.) Mayta \& Molinari, comb. nov.

Basionym: Loricaria ollgaardii M.O. Dillon \& Sagást., Phytologia 59: 228. 1986.

Type: Ecuador, Loja: Amaluza-Palanda, western slope, near the pass (at Lagunas Arreviatadas Pilares), 3350-3450 m, 22 September 1976, B. Øllgaard \& H. Balslev 9687 (F - holotype [photo!]; MO, NY - isotypes). 
Notes. This species is endemic to the wetlands of southern Ecuador, considered Vulnerable (León Yáñez et al. 2011; Ulloa Ulloa et al. 2017). Since the locality is close to the international border, it might be present in Peru as well.

\section{Andicolea pauciflora (Cuatrec.) Mayta \& Molinari, comb. nov.}

Basionym: Loricaria pauciflora Cuatrec., Feddes Repert. Spec. Nov. Regni Veg. 56: 165. 1954.

Type: Ecuador, Zamora Chinchipe: Azuay-Oriente border, eastern Cordillera, between Oña and Yacuambi river, $2900 \mathrm{~m}$, W.H. Camp P-187 (NY - holotype; NL - isotype [photo!]).

Notes. Native in southern Ecuador (Ulloa Ulloa et al. 2017). Rodríguez Rodríguez et al. (2016) cite the species for La Libertad department, in northern Peru.

Andicolea scolopendra (Hook.) Mayta \& Molinari, comb. nov.

Basionym: Baccharis scopolendra Hook., Icon. Pl. 1: pl. 68. 1836

Type: Ecuador, Tungurahua: summit of the cordillera of Píllaro, Quintinian Andes, 3000 m, April 1834, Jameson 77 ( $\mathrm{K}$ - holotype [photo!]).

$\equiv$ Loricaria scopolendra (Hook.) Kuntze, Revis. Gen. Pl. 1: 352. 1891.

Notes. This species is endemic to the Andes of central Ecuador, considered Vulnerable (León Yáñez et al. 2011; Ulloa Ulloa et al. 2017). Cuatrecasas (1954) cited the type erroneously without date or collector's number.

Andicolea thujoides (Lam.) Mayta \& Molinari, comb. nov.

Basionym: Conyza thujoides Lam., Encycl. [J. Lamarck \& al.] 2(1): 90.1786 (as 'thuyoïdes').

Type: Peru: J. de Jussieu [s.n.] (P - holotype [photo!], isotype [photo!]).

三Loricaria thujoides (Lam.) Sch. Bip., Bonplandia 8: 258. 1860 (as 'thyoides').

三Loricaria stenophylla Wedd., Chlor. Andina 1: 165, 1855.

=Loricaria stuebelii Hieron., Bot. Jahrb. Syst. 21(3): 346, 1895.

Type: Peru, La Libertad (?): Pacasmayo, prope Pishenuñuna, 3200 m, A. Stübel 32 (B - syntype, destroyed). Peru, San Martín (?): Moyobamba, prope Calle Calle, 3400-3600 m, A. Stübel 53 (B - syntype, destroyed. F - isosyntype [photo!]).

= Molina incana Ruiz \& Pav., Syst. Veg. Fl. Peruv. Chil.: 211. 1798.

Type: Peru, Huánuco: Pillao ad Silcai, 1787, Ruiz \& Pavón [s.n.] (MA - holoype [photo!]; B, BC, FI, MA - syntypes).

Notes. We agree with Hind (2004) in considering the different spellings of 'thujoides' as correctable mistakes. Loricaria stuebelii was considered endemic to Peru (Beltrán et al. 2006) until it was synonymized by Rodríguez Rodríguez et al. (2016). In the same work, the authors list this species as endemic, however, it is also found in Colombia (Bernal et al. 2016), Ecuador (Ulloa Ulloa
\& Fernández Fernández 2015) and Bolivia (Jørgensen et al. 2014).

Andicolea thyrsoidea (Cuatrec.) Mayta \& Molinari, comb. et stat. nov.

Basionym: Loricaria thujoides var. thyrsoidea Cuatrec., Feddes Repert. Spec. Nov. Regni Veg. 56: 170. 1954.

Type: Peru, Áncash: near Antacocha, Cerro Colorado, E of Canta, 4000-4100 m, 20 June 1925, F.W. Pennell 14685 (F holotype [photo!]; GH, NY, US - isotypes).

Notes. Described from specimens found in central Peru, León et al. (2006) report the species from Bolivia, while Ulloa Ulloa et al. (2017) report it also from Colombia.

Andicolea unduaviensis (Cuatrec.) Mayta \& Molinari, comb. nov.

Basionym: Loricaria unduaviensis Cuatrec., Feddes Repert. Spec. Nov. Regni Veg. 56: 170. 1954.

Type: Bolivia, La Paz: Unduavi, 3300 m, October 1885, H. H. Rusby 1565 (NY - holotype [photo!]; NY, US, GH isotypes).

Notes. The species is endemic to Bolivia, with a conservation status of Data Deficient (Ministerio de Medio Ambiente y Agua 2012; Jørgensen et al. 2014; Ulloa Ulloa et al. 2017).

Andicolea sect. Graveolum (Cuatrec.) Mayta \& Molinari, comb. nov.

Basionym: Loricaria sect. Graveolum Feddes Repert. Spec. Nov. Regni Veg. 56: 157. 1954.

Type: Baccharis graveolens Sch. Bip., Bonplandia 4(4): 51. 1856.

Andicolea graveolens (Sch. Bip.) Mayta \& Molinari, comb. nov.

Basionym: Baccharis graveolens Sch. Bip., Bonplandia 4: 51. 1856.

Type: Peru: in Peruviae rupium fissuris Cordill. summ. pro San Antonio, 5100 m, Lechler 1815 (P - holotype [photo!]; isotype).

$\equiv$ Loricaria graveolens (Sch. Bip.) Wedd., Chlor. Andina: 167. 1856.

Notes. Commonly found in southern Peru, it has been reported from Bolivia (Jørgensen et al. 2014; Ulloa Ulloa et al. 2017).

Andicolea sect. Terminalia (Sch. Bip.) Mayta \& Molinari, comb. nov.

Basionym: Loricaria sect. Terminalia Sch. Bip., Bonplandia 8: 259.1860

Type: Molina ferruginea Ruiz \& Pav., Syst. Veg. Fl. Peruv. Chil. 1: 211. 1798.

Andicolea antisanensis (Cuatrec.) Mayta \& Molinari, comb. nov.

Basionym: Loricaria antisanensis Cuatrec., Feddes Repert. Spec. Nov. Regni Veg. 56: 157. 1954. 
Type: Ecuador, Napo: from Antisana near the snow limit, collected at $4000 \mathrm{~m}$, Jameson 1749 (US - holotype [photo!]).

Notes. Endemic to Ecuador, evaluated as Near Threatened (León Yánez et al. 2011).

Andicolea colombiana (Cuatrec.) Mayta \& Molinari, comb. nov.

Basionym: Loricaria colombiana Cuatrec., Trab. Mus. Nac. Ci. Nat., Ser. Bot. 29: 33. 1935.

Type: Colombia, Tolima: Nevado de Tolima, Cordillera Central, 4300 m, 15 June 1932, Cuatrecasas 2947 (MA - holotype; $\mathrm{F}$ - isotype [photo!]).

Note. The species is found exclusively in Colombia (Bernal et al. 2016; Ulloa Ulloa et al. 2017). In the Field Museum of Natural History database, a specimen (M.T. Dawe 757) with no photograph is filed under 'Loricaria colombiana f. elongata'. It also appears in Missouri Botanical Garden's Tropicos, where it is attributed to Cuatrecasas. We have been unable to find any reference to this or any other subordinate taxon for this species whatsoever.

Andicolea ferruginea (Ruiz \& Pav.) Mayta \& Molinari, comb. nov.

Basionym: Molina ferruginea Ruiz \& Pav., Syst. Veg. Fl. Peruv. Chil. 1: 211. 1798.

Type: Peru, Lima or Junín : Cordilleras et Punas per Tarmae, Cantae et Huarocheri Provincias, Ruiz \& Pavón [s.n.] (MA holotype; BM, FI, G, P - syntypes [photo!]).

$\equiv$ Loricaria ferruginea (Ruiz \& Pav.) Wedd. Chlor. Andina 1: 166.1855.

$=$ Loricaria anceps (Sch. Bip.) Sch. Bip., Bonplandia 8: 259. 1860.

Type: Peru: Haenke [s.n.] (P - holotype [photo!]).

$=$ Loricaria stenophylla var. vernicosa Wedd., Chlor. Andina 1: 165.1856

Type: Peru: Dombey [s.n.] (P - holotype [photo!]).

Notes. Originally thought endemic to Peru, reported also from Ecuador (León et al. 2006; Ulloa Ulloa et al. 2017).

Andicolea ilinissae (Benth.) Mayta \& Molinari, comb. nov.

Basionym: Baccharis ilinissae Benth., P1. Hartw. 202. 1845.

Type: Ecuador, Cotopaxi: in sands near the perpetual snows of Ilinissa (Iliniza), Hartweg 1114 (K - holotype [photo!]).

$\equiv$ Loricaria ilinissae (Benth.) Cuatrec., Feddes Repert. Spec. Nov. Regni Veg. 56: 162. 1954.

Notes. This Andean species has been reported also from Colombia (Bernal et al. 2016; Ulloa Ulloa et al. 2017).

Andicolea lucida (Cuatrec.) Mayta \& Molinari, comb. nov.

Basionym: Loricaria lucida Cuatrec., Feddes Repert. Spec. Nov. Regni Veg. 56: 163. 1954.

Type: Peru, Huánuco: 15 miles northeast of Huánuco, 4600 m, 12 June 1922, J. F. Macbride \& W. Featherstone 2171 (F - holotype [photo!]; GH, US - isotypes [photo!]).

Notes. This species has been reported recently from Bolivia (León et al. 2006; Jørgensen et al. 2014; Ulloa Ulloa et al. 2017).
Andicolea lycopodinea (Cuatrec.) Mayta \& Molinari, comb. nov.

Basionym: Loricaria lycopodinea Cuatrec., Feddes Repert. Spec. Nov. Regni Veg. 56: 164. 1954.

Type: Peru, Huánuco: San Carlos mines, station 6 miles west of Huallanca, in protected pockets of rock cliffs, $2700 \mathrm{~m}$, 30 September 1922, J. F. Macbride \& W. Featherstone 2471 (F - holotype [photo!]; GH, US - isotypes [photo!]).

Notes. This Peruvian species is considered endemic and of Least Concern, since it has big populations in central and northern Peru (Beltrán et al. 2006).

Andicolea macbridei (Cuatrec.) Mayta \& Molinari, comb. nov.

Basionym: Loricaria macbridei Cuatrec., Feddes Repert. Spec. Nov. Regni Veg. 56: 164. 1954.

Type: Peru, Junín: San José, Granitic crevices, 4000 m, 10 June 1922, J. F. Macbride \& W. Featherstone 1093 (F holotype [photo!]; GH, US - isotypes [photo!]).

Notes. This Peruvian species was reported from Bolivia by León et al. (2006) but is only reported from Peru by Ulloa Ulloa et al. (2017).

Andicolea puracensis (Cuatrec.) Mayta \& Molinari, comb. nov.

Basionym: Loricaria puracensis Cuatrec., Feddes Repert. Spec. Nov. Regni Veg. 56: 166. 1954.

Type: Colombia, Cauca: páramo del Puracé, 'El reventao', 3600 m, 11 July 1939, E. Pérez Arbeláez \& J. Cuatrecasas 5922 (US - holotype [photo!]; COL, F - isotype).

Notes. The species has been reported only from the Colombian paramos (Bernal et al. 2016; Ulloa Ulloa et al. 2017).

\section{References}

Agardh, C. A. 1821. Species algarum rite cognitae, cum synonymis, differentiis specificis et descriptionibus succinctis. Volumen primum. Pars prima. Officina Berlingiana, Lund. https://doi.org/10.5962/ bhl.title.45326

Beltrán, H., Granda, A., León, B., Sagástegui, A., Sánchez, I. \& Zapata, M. 2006. Asteraceae endémicas del Perú. Revista Peruana de Biología 13(2): 64s-164s. https://doi.org/10.15381/rpb.v13i2.1807

Bernal, R., Gradstein, S. R. \& Celis, M. 2016. Catálogo de Plantas y Liquenes de Colombia. Universidad Nacional de Colombia, Bogotá.

Bory de Saint-Vincent, J. P. 1825. Dictionnaire classique d'histoire naturelle. Tome Septiéme. Rey et Gravier and Badouin Frères, Paris.

Cuatrecasas, J. 1954. Synopsis der Gattung Loricaria Wedd. Feddes Repertorium Specierum Novarum Regni Vegetabilis 56: 149-172. https://doi.org/10.1002/fedr.19540560204

Dillon, M. D. \& Sagástegui-Alva, A. 1986. New species and status changes in Andean Inulae (Asteraceae). Phytologia 59: 227-233. https://doi.org/10.5962/bhl.part.2767

Flann C. (ed.) 2020. Global Compositae Database. [Accessed May 2021]. https://www.compositae.org/aphia.php.

Hind, D. J. N. 2004. A New Species of Loricaria (Compositae: Inuleae sensu lato) from Ecuador. Kew Bulletin 59: 541-545. https://doi. org/10.2307/4110908 
Jørgensen, P. M., Nee, M. H. \& Beck, S. G. 2014. Catálogo de las plantas vasculares de Bolivia. Missouri Botanical Garden Press, Saint Louis.

Lamouroux, J. V. F. 1813. Essai sur les genres de la famille des Thalassiophytes non articulées. Annales du Muséum d'Histoire Naturelle 20: 21-47, 115-139, 267-293.

León, B. 2002. Significance of August Weberbauer's plant collecting for today's Río Abiseo National Park, northern Peru. Taxon 51: 167-170. https://doi.org/10.2307/1554975

León, B., Pitman, N. \& Roque, J. 2006. Introducción a las plantas endémicas del Perú. Revista Peruana de Biología 13(2): 9s-22s. https://doi.org/10.15381/rpb.v13i2.1782

León Yánez, S., Valencia Reyes, R., Pitman, N. C. A., Endara, C. L., Ulloa Ulloa, C. \& Navarrete, H. 2011. Libro Rojo de las Plantas Endémicas del Ecuador, $2^{a}$ edición. Pontificia Universidad Católica del Ecuador, Quito.

Ministerio de Medio Ambiente y Agua. 2012. Libro Rojo de la Flora Amenazada de Bolivia. Volumen I - Zona Andina. Ministerio de Medio Ambiente y Agua, La Paz.

Neill, D. A. \& Ulloa Ulloa, C. 2011. Adiciones a la Flora del Ecuador: Segundo Suplemento, 2005-2010. Fundación Jatun Sacha, Quito.

Rangel Churio, J. O. 2000. Colombia. Diversidad biótica III. La región de vida paramuna. Universidad Nacional de Colombia and Instituto de Investigación de Recursos Biológicos Alexander von Humboldt, Bogotá.

Rodríguez Rodríguez, E. F., Alvítez Izquierdo, E., Pollack Velásquez, L., Melgarejo Salas, N. \& Sagástegui Alva, A. 2016. Catálogo de
Asteraceae de la región La Libertad, Perú. Sagasteguiana 4(2): 73-116.

Smissen, R. D., Bayer, R. J., Bergh, N. G., Breitwieser, I., Freire, S. E., Galbany-Casals, M., Schmidt-Lebuhn, A. N. \& Ward, J. M. 2020. A revised subtribal classification of Gnaphalieae (Asteraceae). Taxon 69: 778-806. https://doi.org/10.1002/tax.12294

Stackhouse, J. 1809. Tentamen marino-cryptogamicum, ordinem novum; in genera et species distributum, in Classe XXIVta Linnaei sistens. Mémoires de la Société Imperiale des Naturalistes de Moscou 2: 50-97.

Ulloa Ulloa, C. \& Neill, D. A. 2005. Cinco Años de Adiciones a la Flora del Ecuador. Editorial UTPL, Loja.

Ulloa Ulloa, C. \& Fernández Fernández, D. (eds.) 2015. Plantas de los páramos del Distrito Metropolitano de Quito, Ecuador. Museo Ecuatoriano de Ciencias Naturales del Instituto Nacional de Biodiversidad, Quito.

Ulloa Ulloa, C., Acevedo-Rodríguez, P., Beck, S. G., Belgrano, M. J., Bernal, R., P. E. Berry, Brako, L., Celis, M., Davidse, G., Gradstein, S. R., Hokche, O., León, B., León-Yánez, S., Magill, R. E., Neill, D. A., Nee, M. H., Raven, P. H., Stimmel, H., Strong, M. T., Villaseñor Ríos, J. L., Zarucchi, J. L., Zuloaga, F. O. \& Jørgensen, P. M. 2017. An integrated assessment of vascular plants species of the Americas. Science 358: 1614-1617. https://doi.org/10.1126/ science.aao0398

Weddell, H. A. 1855. Chloris andina: essai d'une flore de la región alpine des Cordillères de l'Amérique du Sud. Tome Premier. P. Bertrand, Paris. https://doi.org/10.5962/bhl.title.217 